\title{
Future of minimally invasive surgery in temporomandibular joint pathology
}

\author{
Florencio Monje \\ Department of Oral and Maxillofacial Surgery, University Hospital of Badajoz, Badajoz 06001, Spain.
}

Correspondence to: Dr. Florencio Monje, Department of Oral and Maxillofacial Surgery, University Hospital of Badajoz, Badajoz 06001, Spain. E-mail: fmonje@oralmaxilofacial.com

How to cite this article: Monje F. Future of minimally invasive surgery in temporomandibular joint pathology. Stomatological Dis Sci2020;4:2. http://dx.doi.org/10.20517/2573-0002.2019.11

Received: 13 May 2019 Accepted: 6 Aug 2019 Revised: 8 May 2020 Accepted: 8 May 2020 Published: 30 Jun 2020

Science Editors: Raúl González-García Copy Editor: Jing-Wen Zhang Production Editor: Tian Zhang

\begin{abstract}
Minimally invasive surgery (MIS) has experienced a surge in popularity over the past few decades. The main reason for this is the significant reduction of surgical trauma with the minimisation or even elimination of incisions. Temporomandibular joint pathology remains poorly understood and treatment is controversial. For this reason, application of MIS to temporomandibular joints (TMJ-MIS) could be an option for patients with failure of conservative management and where open treatment could be considered aggressive although TMJ-MIS itself is also considered controversial. In this paper, we will examine the different aspects of developing TMJ-MIS in the future: biology and diagnosis, diagnostic arthroscopy, surgical technique, knowledge and training, and different treatments.
\end{abstract}

Keywords: Temporomandibular joint, temporomandibular joint surgery, temporomandibular joint arthroscopy, minimally invasive surgery

\section{INTRODUCTION}

Minimally invasive surgery (MIS) has experienced a surge in popularity over the past few decades. For a number of operations - such as appendectomy, tubal ligation, cholecystectomy, gastric bypass, myomectomy and prostatectomy - more than $90 \%$ are now performed through MIS approaches. The main reason behind this paradigm shift is the significant reduction of trauma to the patient's body that results from the minimization or even elimination of surgical incisions. This reduction in physical trauma, in turn, leads to a number of additional benefits for the patient such as a lower incidence of complications, reduced pain, quicker recovery, shorter length of hospital stay, minimal cosmetic deformity, decreased psychological impact and overall improved quality of life. Choosing the MIS approach over open surgery however, also

cc) (7) The Author(s) 2020. Open Access This article is licensed under a Creative Commons Attribution 4.0 sharing, adaptation, distribution and reproduction in any medium or format, for any purpose, even commercially, as long as you give appropriate credit to the original author(s) and the source, provide a link to the Creative Commons license, and indicate if changes were made.

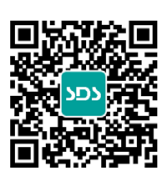


means embracing a series of potential disadvantages from the surgeon's perspective. These include limited operating spaces, lack of haptic feedback, loss of stereovision and depth perception, diminished hand-eye coordination, prolonged learning curves, extensive operative times and increased costs ${ }^{[1]}$.

There are however, various different issues that characterize temporomandibular joints (TMJ) pathology including etiology and pathophysiology, controversies about appropriate treatment, and even more debate with regard to treatment through $\mathrm{MIS}^{[2]}$.

In addition to joint replacement and internal fixation of fractures, arthroscopic surgery is regarded as one of the three greatest improvements in the treatment of patients with conditions affecting the musculoskeletal system during the twentieth century ${ }^{[3]}$.

TMJ arthroscopy is the MIS approach to this joint. The future of TMJ-MIS could be examined through: biology and diagnosis, diagnostic arthroscopy, simplifying surgical technique, knowledge and training, treatments.

\section{BIOLOGY AND DIAGNOSIS}

The literature is lacking concerning the correlation between clinical symptoms and arthroscopic findings of TMJ pathology. For example, the relation of TMJ synovitis with pain is still controversial. While Murakami et al. ${ }^{[4]}$ finds a weak relation, Holmlund and Axelsson ${ }^{[5]}$ finds no association at all. The type of pathology however, can influence arthrosopic findings and some observed correlations. For example, if arthroscopic exploration is undertaken in patients with rheumatoid arthritis, correlation will indeed be found between pain and the degree of synovitis ${ }^{[6]}$. Sato et al. ${ }^{[7]}$ though found a positive correlation between pain symptoms and the degree of synovitis and an inversely proportional relationship between mouth opening and adhesions. More studies on the interpretation of and relationship between symptoms and prognosis of patients with TMJ pathology are needed.

It is recognized that different chemical mediators may be involved in producing symptoms. In general, inflammatory cytokines are highly expressed in TMJs affected by synovitis ${ }^{[8]}$. Proteomics, which studies the entire set of proteins, such as inflammatory mediators that are produced or modified by an organism or system, will be important in rheumatoid disease and it has much to inform knowledge on TMJ pathology ${ }^{[9]}$.

Another point for further discussion in the diagnosis of TMJ disorders would be the value of cone beam computerized tomography. Cone beam computerized tomography is not routine used but its sensitivity in the diagnosis of sclerosis and condylar changes, and its relationship with chondromalacia is higher compared to $\mathrm{MRI}^{[10]}$.

Obviously, magnetic resonance imaging will be important for information about the different characteristics of the soft and hard tissues of the $\mathrm{TMJ}^{[11]}$. We believe that other radiological investigations that are more accessible, less expensive and less difficult to interpret than magnetic resonance imaging should be considered. For example, high-resolution ultrasound enables dynamic examination which can aid in making clinical decisions but it is highly operator dependent ${ }^{[12,13]}$.

Although we have gained some insight into the relationship between clinical symptoms, radiological findings and arthroscopic lesions, more universal and affordable diagnostic tools are required for TMJ pathology.

\section{DIAGNOSTIC ARTHROSCOPY}

When exploring the TMJ, we cannot forget to explore both the superior and inferior joint spaces. Liedberg et al. ${ }^{[14]}$ evaluated the diagnostic accuracy of upper compartment arthroscopy of the TMJ. An 
arthroscopic diagnosis of pathological changes seems to be reliable (high specificity), but normal findings include a substantial risk of under-diagnosing pathology (low sensitivity). Tzanidakis et al. ${ }^{[15]}$ studied arthroscopy and surgery 6 months post-arthroscopy. Overall, arthroscopy had 87\% sensitivity and 99\% specificity in diagnosing diseases of the TMJ. In summary, the specificity of arthroscopy is higher than its sensitivity since normal findings on arthroscopy include a substantial risk of under-diagnosis.

Inexperienced or novice TMJ surgeons tend to enter the inferior articular space accidentally. Classically, it is considered inadvisable to enter this space due to potential damage to the cartilage of the mandibular condyle. At this location, there may be pathological findings such as synovitis or adhesions. Since jaw opening consists of pure rotation of the condyle over the disk, persistence of symptoms in patients treated with TMJ arthroscopy may be the result of misdiagnosis with untreated adhesions and synovitis within the inferior joint compartment ${ }^{[16]}$.

Besides arthroscopy, it would be very useful if other methods can aid in the analysis of surfaces or subsurfaces of the tissues involved in TMJ pathology. Ultrasound was performed on human cadavers, in combination with traditional TMJ arthroscopy. During these investigations, the main articular structures were identified: the glenoid fossa of the temporal bone, articular disc, mandibular condyle, and retrodiscal tissue. The combination of arthroscopy and ultrasound imaging provides more information on the position, movement, and pathological changes in joint structures of the TM ${ }^{[17]}$.

Bioelectrical impedance analysis is an easy-to-use, non-invasive method that measures the electrical properties of the patient's tissues. Classic bioimpedance devices like body composition analyzers are commercially available on the market. In recent years, many new applications are finding their way into medical practice ${ }^{[18,19]}$. In future, this method could be used through the arthroscope to assess the different characteristics of tissues in both compartments of the TMJ.

Standard light provides a poor contrast between the collagenous joint structures (as they all appear offwhite). Additionally, degeneration of joint structures further hampers the contrast and visualization. Collagen though, is an autofluorescent tissue component which can emit blue/green light (emission $400-405 \mathrm{~nm}$ ) after excitation with violet/blue light (at $325 \mathrm{~nm}$ ). This autofluorescence property is already in use by Orthopedics in other joints such as the shoulder ${ }^{[20]}$ and could be applied to TMJ arthroscopy.

We need devices that can accurately evaluate all the tissues and compartments of the TMJ to assist us in decision making.

\section{SIMPLIFYING SURGICAL TECHNIQUE}

In most modern setups, high-resolution CCD cameras are used instead to transmit a live video stream to a flat screen. One of the main problems associated with video-assisted MIS is the loss of stereopsis i.e. perception of depth and three-dimensionality. This occurs when a three-dimensional (3D) image is projected on a two-dimensional screen, and is often the cause of impeded hand-eye coordination and erroneous movements of tools. The use of $3 \mathrm{D}$ technology in a small joint such as the TMJ would not be useful at this time because the advantages of this technique are clearer in larger joints.

Therefore, hip arthroscopy has an exploratory role in the treatment of undiagnosed hip or groin pain. Therapeutically, there are now many indications for hip arthroscopy including the removal of loose bodies, trochanteric bursitis and fpr femoroacetabular impingement. Recent navigation systems in hip arthroscopy also offer drastic improvements in the way of information ${ }^{[21]}$. For instance, navigation or computer-assisted TMJ arthroscopy can provide the surgeon with continuously updated information about the puncture sites and the location of instruments in relation to target and critical structures ${ }^{[22]}$. For a joint such as the TMJ however, "using a sledge-hammer to crack a nut" would seem more appropriate. 
With regard to the approach of inserting the second cannula, there are several possible ways. We prefer the simple geometric principle of triangulation. After diagnostic arthroscopy has been completed, the other cannula can be inserted with a sharp and blunt obturator in an anterolateral point and medial direction. Two problems make this technique very difficult: the two compartments of the TMJ are separated by the articular disc, and the articular eminence. In future, computer assisted surgery could be useful in providing augmented reality, an "image-enhanced operating environment". It can be created by overlaying of a visual representation of the subsurface anatomy and critical structures, while integrating it with the video feed from the camera ${ }^{[23]}$. Augmented reality could be useful in different approaches to the TMJ although experimental and cadaver studies have been published. Wang et al. ${ }^{[24]}$ presented a system, in cadavers, to provide augmented reality visualization during TMJ arthrocentesis to increase the precision of skin punctures.

Another point to be developed could be different instruments or technologies for use inside of the TMJ. Once inside the joint, the size of the tools needs to be minimized (to ablate tissue, seal vessels and cauterize). Many years ago, based on the effects of electricity, we developed mono- and bipolar cautery ${ }^{[25]}$, excellent tools but without clear visualization and have important effects on surrounding tissues to coagulate and cut. Some years ago, radiofrequency emerged with less thermal damage to surrounding tissues, no smoke or bubbling, and greater treatment accuracy ${ }^{[26]}$ and we now have the plasma sprayer system for surgeries. Plasma is composed of highly ionized particles. These ionized particles separate molecules from each other and reduce tissue volume. It will not damage tissues directly and cause little damage to surrounding healthy tissues ${ }^{[27]}$. It could be key for the next step required in arthroscopy resection. Sometimes, lasers are also used for ablating tissues but it is, and will be expensive with no real advantages otherwise.

Another fertile area of research in surgical robotics is the application of machine learning algorithms for the creation of human-robot cooperative control frameworks in which the surgeon is aided by the machine in the most critical parts of a task. Using a double console setup can thus be beneficial for training and learning purposes, both for the novice and the machine: as expert surgeons perform a physical or virtual surgical simulation, trainees can follow their steps using another machine, while the robot can track the instruments, implementing complex algorithms in order to improve performance. The surgical community is still focused on the question of 'robotics' in surgery. To be clear, the term "robot" is completely inadequate to describe a da Vinci: telemanipulation systems. In robotic-assisted surgery, the instruments are not moved directly by the surgeon as he operates through master controls, which improves the surgeon's dexterity and skillfulness leading to ultraprecision with no tremors at all. The advantages though seem to be clinically significant only in a subset of surgical procedures, such as rectal resection, abdominal surgery, cardiac surgery or some ENT procedures. It is important to understand however, that because of the size of the hardware, its use is limited in joints and even less in the TMJ. A major area of focus in the development of surgical robotic platforms is the implementation of haptic feedback. Haptics include sensations such as force, pressure, temperature and texture - all qualities that are difficult to quantify and represent in robotic and MIS procedures. It is hence a challenge for the surgeon to judge the right amount of force to apply and to make decisions based on tactile palpation. Another major challenge is the cost with economic analyses demonstrating cost-effectiveness only for high-volume centers ${ }^{[28,29]}$. In other words, robotics is far from being used in TMJ.

\section{KNOWLEDGE AND TRAINING}

The therapeutic results of TMJ-MIS could be related to patient selection, choice of technique and quality of MIS-arthroscopy but there is a high initial learning curve in MIS.

Traditionally, surgeons have acquired their skills by operating on patients under supervision with gradual independence (the so-called apprentice system or Halsted model). This method consists of "observing, 
practicing, and teaching". Whereas some open surgical skills may be innate, which develop naturally because of lifelong experiences with basic tools and utensils, the skills needed to perform arthroscopy are not naturally derived, and compared to open surgery, arthroscopy requires specific operative skills. For example, arthroscopy requires surgeons to perceive a three-dimensional environment from a two-dimensional camera image. Furthermore, specific hand-eye coordination is required, and the sense of touch is minimal. Finally, every endoscopic procedure poses the challenge of the fulcrum effect, such that when the surgeon moves his hand to the right, the working end of the instrument moves to the left on the monitor and vice versa. The surgeon has to actively compensate for this effect.

Cadaver studies continue to be the gold standard due to close resemblance with real patients. However, there are certain drawbacks, such as the high costs, legal requirements, lack of reusability, failure to reproduce different pathologies, and numerous political, cultural, and religious considerations. While surgeons have to learn from their mistakes, overcoming the learning curve should preferably be done outside of the surgical room.

Different simulators could be used: (1) box trainers are devices where trainees have to perform tasks using real instruments in a box while watching their movements on a video screen. They are relatively cheap compared to more advanced simulation models and the instruments used are the same as those in the operating environment. The disadvantages are they lack realistic features and the tasks do not sufficiently resemble those in real-life; (2) realistic simulators by recreating actual scenarios. The first prototype of a physical simulator designed for training in TMJ arthroscopy was presented. It was constructed according to anthropometric standards using a material that reproduces the different textures and colors of all anatomical parts. One of the advantages provided by the use of simulators is the possibility of repeating the exercise (reusability) as many times as needed to acquire the basic skills necessary ${ }^{[31]}$; and (3) virtual reality (VR) trainers are computer-based applications that allow for movement in free space whilst performing tasks in a virtual operative environment. VR can be combined with a physical model as an "overlay", hence they are often referred to as augmented reality trainers. These types of simulators offer the advantages of both systems, namely haptic feedback, real-life surgical material, realistic internal views, and training of different scenarios. For training in arthroscopic skills, knee and shoulder arthroscopy simulators based on VR are of interest ${ }^{[32]}$.

\section{TREATMENT}

MIS is composed of three achievements: diagnosis, surgical resection and reconstruction. Different methods of suturing have been described ${ }^{[33-36]}$ and a number of techniques have been devised for this purpose. Independent of clinical results, there does not seem to be any evidence that surgically repositioning a disc maintains it in a normal position in all cases. We must therefore be cautious when introducing new operative arthroscopic procedures that at best, may provide only marginal benefit over simple lavage and in the worst case, cause more surgical trauma. A successful surgery for the surgeon is not always a successful surgery for the patient. Complicated arthroscopic disc repositioning and suturing might not be considered routine.

Nowadays, mesenchymal stem cells could be considered as the latest "research trend" in the field of biology and medicine and their application in regenerative medicine is growing. Some modalities involve direct implantation of mesenchymal stem cells into the defect site while others use scaffolds to support the cells ${ }^{[37,38]}$. Vapniarsky et al. ${ }^{[39]}$ approached anatomic defects by using scaffold free tissue engineering as an approach for the regeneration of TMJ discs to address disc thinning in the pathogenesis of TMJ degeneration. Treatment of these conditions that are prevalent in TMJ pathologies are palliative and not reparative. To address this, scaffold-free tissue-engineered implants were created using allogeneic, passaged costal chondrocytes. A combination of compressive and bioactive stimulation regimens produced implants 
with mechanical properties akin to those of the native disc. The efficacy in repairing disc thinning was examined in mini pigs. Compared to empty controls, treatment with tissue-engineered implants restored disc integrity.

The healing capacity of bone marrow mesenchymal stem cells (BMMSCs) has been evaluated in various studies but one study aimed to evaluate the effect of BMMSCs on the healing of TMJs with induced rheumatoid arthritis. Rats were treated by intravenous injection of BMMSCs. The results indicate that the treatment of induced rheumatoid arthritis with BMMSCs shows promise that need to be further investigated in humans ${ }^{[40]}$. Thus, the future could be regenerative medicine through tissue engineering in minimally invasive arthroscopy for TMJ pathology.

\section{CONCLUSION}

More studies are needed to interpret the relationship between clinical symptoms and the prognosis in patients with TMJ articular pain. More affordable and universal diagnostic tools are required for use in this pathology. An arthroscopic diagnosis of normality includes a substantial risk of under diagnosis of pathologic changes. We need devices that can accurately diagnose all the tissues and compartments of the TMJ to assist us in the decision making process. New technologies such as navigation and robotics are likely unsuitable for TMJ; however, augmented reality may be of use to develop best techniques. In relation to the steep learning curve in MIS, cadavers continue to be the gold standard but realistic virtual simulation would be the most logical evolution in the training of TMJ-MIS. The future of TMJ-MIS may be through regenerative medicine approaches such as tissue engineering.

\section{DECLARATIONS}

\section{Authors' contributions}

The author contributed solely to the article.

\section{Availability of data and materials}

Not applicable.

\section{Financial support and sponsorship}

None.

\section{Conflicts of interest}

The author declared that there are no conflicts of interest.

\section{Ethical approval and consent to participate}

Not applicable.

\section{Consent for publication}

Not applicable.

\section{Copyright}

(c) The Author(s) 2020.

\section{REFERENCES}

1. Antoniou SA, Antoniou GA, Antoniou AI, Granderath FA. Past, present, and future of minimally invasive abdominal surgery. JSLS 2015;19:e2015.00052.

2. González-García R. The current role and the future of minimally invasive temporomandibular joint surgery. Oral Maxillofac Surg Clin North Am 2015;27:69-84.

3. Siemieniuk RAC, Harris IA, Agoritsas T, Poolman RW, Brignardello-Petersen R, et al. Arthroscopic surgery for degenerative knee 
arthritis and meniscal tears: a clinical practice guideline. BMJ 2017;357:j1982.

4. Murakami K, Segami N, Fujimura K, Iizuka T. Correlation between pain and synovitis in patients with internal derangement of the temporomandibular joint. J Oral Maxillofac Surg 1991;49:1159-61; discussion 1162.

5. Holmlund AB, Axelsson S. Temporomandibular arthropathy: correlation between clinical signs and symptoms and arthroscopic findings. Int J Oral Maxillofac Surg 1996;25:178-81.

6. Gynther GW, Holmlund AB, Reinholt FP, Lindblad S. Temporomandibular joint involvement in generalized osteoarthritis and rheumatoid arthritis: a clinical, arthroscopic, histologic, and immunohistochemical study. Int J Oral Maxillofac Surg 1997;26:10-6.

7. Sato FRL, Lima CAA, Tralli G, da Silva RA. Is there a correlation between arthroscopic findings and the clinical signs and symptoms of patients with internal derangement of the temporomandibular joint? A prospective study. Int J Oral Maxillofac Surg 2019;48:233-8.

8. Monje-Gil F, Nitzan D, González-Garcia R. Temporomandibular joint arthrocentesis. Review of the literature. Med Oral Patol Oral Cir Bucal 2012;17:e575-81.

9. Papadaki M, Rinotas V, Violitzi F, Thireou T, Panayotou G, et al. New insights for RANKL as a proinflammatory modulator in modeled inflammatory arthritis. Front Immunol 2019;10:97.

10. Larheim TA, Hol C, Ottersen MK, Mork-Knutsen BB, Arvidsson LZ. The role of imaging in the diagnosis of temporomandibular joint pathology. Oral Maxillofac Surg Clin North Am 2018;30:239-49.

11. Krohn S, Joseph AA, Voit D, Michaelis T, Merboldt KD, et al. Multi-slice real-time MRI of temporomandibular joint dynamics. Dentomaxillofac Radiol 2018;20:20180162.

12. Kalyan S. The role of ultrasound in diagnosis of temporomandibular joint disc displacement: a case-control study. J Maxillofac Oral Surg 2018; 17:383-8.

13. Yilmaz D, Kamburoğlu K. Comparison of the effectiveness of high resolution ultrasound with MRI in patients with temporomandibular joint disorders. Dentomaxillofac Radiol 2019;48:20180349.

14. Liedberg J, Westesson PL. Diagnostic accuracy of upper compartment arthroscopy of the temporomandibular joint: correlation with postmortem morphology. Oral Surg Oral Med Oral Pathol 1986;62:618-24.

15. Tzanidakis K, Sidebottom AJ. How accurate is arthroscopy of the temporomandibular joint? A comparison of findings in patients who had open operations after arthroscopic management failed. Br J Oral Maxillofac Surg 2013;51:968-70.

16. González-García R, Moreno-Sánchez M, Moreno-García C, Román-Romero L, Monje F. Arthroscopy of the inferior compartment of the temporomandibular joint: a new perspective. J Maxillofac Oral Surg 2018;17:228-32.

17. Patonay L, Nagy K, Engelke W. Real-time endoarticular ultrasound imaging of the TMJ--a new diagnostic possibility? A cadaver study. Int J Oral Maxillofac Surg 2002;31:553-7.

18. Mazzoni MB, Perri A, Plebani AM, Ferrari S, Amelio G, et al. Electrical impedance tomography in children with community acquired pneumonia: preliminary data. Respir Med 2017;130:9-12.

19. Axelsson L, Silander E, Bosaeus I, Hammerlid E. Bioelectrical phase angle at diagnosis as a prognostic factor for survival in advanced head and neck cancer. Eur Arch Otorhinolaryngol 2018;275:2379-86.

20. Nguyen DT, van Horssen P, Derriks H, van de Giessen M, van Leeuwen T. Autofluorescence imaging for improved visualization of joint structures during arthroscopic surgery. J Exp Orthop 2017;4:19.

21. Tannenbaum EP, Ross JR, Bedi A. Pros, cons, and future possibilities for use of computer navigation in hip arthroscopy. Sports Med Arthrosc Rev 2014;22:e33-41.

22. Wagner A, Undt G, Watzinger F, Wanschitz F, Schicho K, et al. Principles of computer-assisted arthroscopy of the temporomandibular joint with optoelectronic tracking technology. Oral Surg Oral Med Oral Pathol Oral Radiol Endod 2001;92:30-7.

23. Feußner H, Rau B. Augmented reality, cyber-physical systems and robotics: nice to have or a program with future? Visc Med 2018;34:8-9.

24. Wang YY, Liu HP, Hsiao FL, Kumar A. Augmented reality for temporomandibular joint arthrocentesis: a cadaver study. Int J Oral Maxillofac Surg 2019;48:1084-7.

25. Tarro AW. TMJ arthroscopic diagnosis and surgery: clinical experience with 152 procedures over a 2 1/2-year period. Cranio 1991;9:10719.

26. Monje F, Chossegros C. Therapeuthic Arthroscopy of the Temporomandibular Joint. Retrodiscal Tissue Coagulation. En Surgical magement of the Temporomandibular Joint. Vol 1: Arthroscopy. I Tunes Store, 2014

27. Enochson L, Sönnergren HK, Mandalia VI, Lindhal A. Bipolar Radiofrequency Plasma Ablation indices proliferation and alters cytokine expression in Human articular cartilage chondrocytes. Arthroscopy 2012;28:1275-82.

28. Goradia V. Computer-assisted and robotic surgery in orthopedics:where we are in 2014. Sports Med Arthrosc Rev 2014;22:202-5.

29. Tonutti M, Elson DS, Yang GZ, Darzi AW, Sodergren MH. The role of technology in minimally invasive surgery: state of the art, recent developments and future directions. Postgrad Med J 2017;93:159-67.

30. Wittich CM, Agrawal A, Cook DA, Halvorsen AJ, Mandrekar JN, et al. E-learning in graduate medical education: survey of residency program directors. BMC Med Educ 2017;17:114-9.

31. Monje Gil F, Hernandez Vila C, Moyano Cuevas JL, Lyra M, Pagador JB, et al. Validation of a simulator for temporomandibular joint arthroscopy. Int J Oral Maxillofac Surg 2016;45:836-41.

32. Yari SS, Jandhyala CK, Sharareh B, Athiviraham A, Shybut TB. Efficacy of a virtual arthroscopic simulator for orthopaedic surgery residents by year in training. Orthop J Sports Med 2018;6:2325967118810176.

33. McCain JP, Podrasky AE, Zabiegalski NA. Arthroscopic Disc Repositioning and suturing: a preliminary report. J Oral Maxillofac Surg 1992;50:568-79.

34. Goizueta CC, Muñoz M. The posterior double pass suture in repositioning of the temporomandibular disc during arthroscopic surgery: a 
reprint of 16 cases. J Craniomaxillofac Surg 2012;40:86-93.

35. Yang C, Cai XY, Chen MJ, Zhang SY. New arthroscopic disc repositioning and suturing technique for treating an anteriorly displaced disc of the temporomandibular joint: part I--technique introduction. Int J Oral Maxillofac Surg 2012;41:1058-63.

36. Goizueta C, Rodriguez-Campo FJ, Monje F. Therapeutic arthroscopy of the temporomandibular joint. Other discal sutures (II). En Surgical magement of the Temporomandibular Joint. Vol 1: Arthroscopy. I Tunes Store, 2014.

37. Koh YG, Kwon OR, Kim YS, Choi YJ, Tak DH. Adipose-derived mesenchymal stemcells with microfracture versus microfracture alone: 2-year follow-up of a prospective randomized trial. Arthroscopy 2016;32:97-109.

38. Acri TM, Shin K, Seol D, Laird NZ, Song I, et al. Tissue engineering for the temporomandibular joint. Adv Healthc Mater 2019;8:e1801236.

39. Vapniarsky N, Huwe LW, Arzi B, Houghton MK, Wong ME, et al. Tissue engineering toward temporomandibular joint disc regeneration. Sci Transl Med 2018; 10:eaaq1802.

40. El Qashty RMN, Mohamed NN, Radwan LRS, Ibrahim FMM. Effect of bone marrow mesenchymal stem cells on healing of temporomandibular joints in rats with induced rheumatoid arthritis. Eur J Oral Sci 2018;126:272-81. 